\title{
Prostatic hyperplasia in acromegaly, a myth or reality: a case-control study
}

\author{
Santosh Kumar', Ram Niwas Yadav², Prakamya Gupta ${ }^{3}$, Balan Louis Gaspar ${ }^{4}$, \\ Nandita Kakkar, Alka Verma4, Girish Parthan ${ }^{5}$, Anil Bhansali5 ${ }^{5}$, K K Mukherjee ${ }^{3}$, \\ Márta Korbonits ${ }^{6}$ and Pinaki Dutta ${ }^{5}$ \\ Departments of ${ }^{1}$ Urology, ${ }^{2}$ General Surgery, ${ }^{3}$ Neurosurgery, ${ }^{4}$ Histopathology and ${ }^{5}$ Endocrinology, Postgraduate \\ Institute of Medical Education and Research, 4th Floor, F Block, Nehru Hospital, Chandigarh 160012, India and \\ ${ }^{6}$ Barts and the London School of Medicine, William Harvey Research Institute, Queen Mary University, London \\ EC1M6BQ, UK
}

Correspondence
should be addressed
to P Dutta
Email
pinaki_dutta@hotmail.com

\begin{abstract}
Context: Disorders of the prostate gland are more prevalent in patients with acromegaly. GH-insulin-like growth factor 1 (IGF1) axis plays an additive role in prostatic growth and development.

Objective: To correlate the structural and histopathological changes of the prostate and prostatic symptoms with GH/IGF1 in patients with acromegaly.

Design: Case-control study, from January 2012 to November 2013.

Setting: Tertiary referral centre university hospital in Northern India.

Patients: Fifty-three men with acromegaly and 50 healthy men matched for age and BMI.

Main outcome measures: International Prostate Symptom Score (IPSS), prostate-specific antigen (PSA) levels, dimensions of the prostate on trans-rectal ultrasonography, parameters on uroflowmetry, and immunopositivity with anti-IGF1 antibody in prostatic tissue biopsies.

Results: Despite low serum testosterone levels $(8.9 \mathrm{nmol} / \mathrm{l} \mathrm{vs} 14.3 \mathrm{nmol} / \mathrm{l}$, acromegaly vs control), patients with acromegaly had marginally higher IPSS, PSA levels, and grades of enlarged prostate and obstructive features on uroflowmetry compared with controls. Dimensions of the prostate on ultrasonography were also significantly higher in patients. These changes were present irrespective of age, current gonadal status, and disease activity. Evidence of prostatic hyperplasia on biopsy was seen in six of 14 patients $(42.8 \%)$ who underwent prostatic biopsy while it was absent in the controls. Immunohistochemistry with anti-IGF1 antibody showed moderate positivity in all the 14 patients who underwent biopsy with benign prostatic hyperplasia, compared with mild positivity in $21 \%$ of controls. Similarly, 14 control patients with prostatic malignancies showed variable positivity, four patients had strong, two each had mild and moderate positivity, while six were negative. Conclusions: In patients with acromegaly, there is a higher frequency of structural changes in the prostate, along with greater prostatic volume and obstructive features, compared with healthy controls, irrespective of age, gonadal status, and disease activity.
\end{abstract}

\section{Introduction}

Acromegaly is caused by the excessive secretion of growth hormone (GH). Testosterone and dihydrotestosterone (DHT) are considered to be the major regulators of prostate growth and function $(1,2)$. However, in recent years, insulin-like growth factor 1 (IGF1), IGF2, and IGF-binding proteins (IGFBPs) have been shown to have a pivotal role in the growth and development of prostate in normal physiology as well as in pathological states $(3,4,5,6,7)$. Both IGF1 and IGF2 have direct mitogenic effects on prostatic epithelial cells and have been implicated in the
(C) 2015 European Society of Endocrinology Printed in Great Britain
Published by Bioscientifica Ltd. 
pathogenesis of prostate cancer, but their actions are still unclear (7). Common causes of mortality and morbidity in patients with acromegaly are cardiovascular, respiratory, and neoplastic disorders (8). A few studies have reported a higher prevalence of prostate disorders in patients with acromegaly compared with age-matched healthy controls $(3,4,5,6)$. Despite the higher prevalence of 'prostatic enlargement' and possibly an increased risk of prostatic cancer in patients with acromegaly, no guidelines recommend screening for prostatic disorders, at baseline or during follow-up. In this study, we evaluated and correlated prostatic symptoms using International Prostate Symptom Score (IPSS) and urodynamic studies with structural and histopathological changes in the expression of IGF1 in the prostate gland for the first time, in relation to GH/IGF1 axis in patients with acromegaly. This topic is important given the increasing interest in GH/IGF1 axis and the influence of acromegaly on increased risk of benign prostatic hyperplasia (BPH).

\section{Subjects and methods}

This case-control study was conducted between January 2012 and November 2013 at the Postgraduate Institute of Medical Education and Research, Chandigarh, India. All patients gave written informed consent and the study was approved by the Institute's Ethics Committee. Fifty-three males aged 20-60 years, with a diagnosis of acromegaly, were enrolled for the study. Patients with a history of urogenital disorders such as urethral strictures, bladder stones, meatal stenosis, neurogenic bladder, and any known prostatic disorder or those with a history of transurethral resection of prostate were excluded from the study. In addition, patients with diabetes mellitus of more than 10 years of duration (as they may be likely to have cystopathic changes), those with postoperative diabetes insipidus, and patients on octreotide LAR treatment (as somatostatin receptor subtypes 1 and 2 are found in stromal cells of benign and malignant prostate tissues) were also excluded. Fifty healthy subjects whose age, BMI, and educational status matched served as controls. All patients were replaced with a stable dose of respective hormone deficiencies at least 3 months before enrollment in the study.

All patients had a GH-secreting pituitary macroadenoma, six patients additionally had hyperprolactinemia with immunostaining positivity for prolactin. Patients were divided into those having active or inactive disease based on the GH response to $75 \mathrm{~g}$ oral glucose challenge and age- and gender-specific IGF1 levels. Patients were further divided into hypogonad or eugonad based on morning serum testosterone levels.

Pituitary target hormones, complete biochemistry, hematological parameters, prostate-specific antigen (PSA), trans-abdominal ultrasonography of the kidneys, ureters, bladder and prostate (KUB), digital rectal examination (DRE), trans-rectal ultrasonography (TRUS) of the prostate, and uroflowmetry were performed in all patients and controls. Prostatic symptoms were evaluated by IPSS. Image-guided trans-rectal prostatic biopsy was done in 14 patients and equal number of controls, who were randomly selected.

The biochemical and hormonal parameters were as follows: serum PSA was performed by solid-phase immunoassay with a detection range of $10.24-25 \mathrm{pg} / \mathrm{ml}$, as described earlier. The normal range for healthy individuals is $0-4 \mathrm{ng} / \mathrm{ml}$ with a $\mathrm{CV}<10 \%$. Hormonal parameters including GH, IGF1, prolactin, luteinizing hormone (LH), follicle-stimulating hormone, testosterone, cortisol, tri-iodothyronine, thyroxine, and thyroid-stimulating hormone were estimated by electrochemiluminescence immunoassay (COBAS, 800, Roche-Hitachi).

Ultrasonography of KUB and TRUS prostate (Philips iU22 xMATRIX) was performed in the Department of Radiology by a single radiologist, who was blinded for the study. The transducers used were 1-5( $\left.\mathrm{C}_{5-1}\right)$ megahertz for KUB and 5-9 $\left(C_{5-9}\right)$ megahertz for TRUS. Before the TRUS, all subjects received enema, and a DRE was performed. The transducer was covered with a disposable rubber sheath and was lubricated with ultrasound transmission gel and gradually inserted about $3 \mathrm{~cm}$ into rectum and then directed toward the anterior rectal wall. Prostate examination included antero-posterior (AP), transversal (TR), and craniocaudal (CC) diameters; the transitional zone, median lobe, morphology of boundaries and the occurrence of calcifications, cysts, and nodules were evaluated. Prostatic volume (PV) and the volume of the transitional zone were calculated by means of the standard ellipsoid formula $(0.52 \times \mathrm{AP}$ diameter $\times \mathrm{TR}$ diameter $\times \mathrm{CC}$ diameter $) . \mathrm{PV}$ exceeding $30 \mathrm{ml}$ was considered hyperplastic prostate (9).

IPSS is based on seven questions concerning urinary symptoms and one question concerning quality of life. Each question about urinary symptoms allows the patient to choose an answer out of six options, indicating increasing severity of a particular symptom. The answers are assigned points from 0 to 5 . The total score can therefore range from 0 to 35 (asymptomatic to very symptomatic, Supplementary Table 1 , see section on supplementary data given at the end of this article). Questions refer to the following urinary symptoms: incomplete emptying, 
frequency, intermittency, urgency, weak stream, straining, and nocturia. The eighth question refers to the patient's perceived quality of life. The first seven questions of the IPSS are identical to the questions appearing on the American Urological Association (AUA) Symptom Index, which currently categorizes symptoms as follows: mild (symptom score $\leq 7$ ), moderate (symptom score range 8-19), and severe (symptom score range 20-35) $(10,11)$. IPSS has been previously validated in our population (Santosh Kumar and SK Singh, unpublished data).

All patients had standard uroflowmetry, which is a diagnostic test in which the speed of urine flow (average and maximum) is measured electronically as millilitres of urine passed per second. TRUS was used to measure postvoid residual (PVR) urine volume, which is the quantity of urine left in the bladder after urination. A PVR of $<50 \mathrm{ml}$ was regarded as adequate bladder emptying.

\section{Prostate biopsy}

A single investigator (S Kumar) performed TRUS-guided sexant prostatic biopsies using an 18-gauge biopsy needle under local anesthesia in 14 patients with acromegaly, who were randomly selected. Equal number of controls also underwent biopsy. Location of prostatic biopsy was both peripheral and transitional zone. Urine culture and coagulation profile were done in all subjects before biopsy and prophylactic antibiotics (ciprofloxacin and amikacin) were given after biopsy for 3 days.

Biopsy specimens were subjected to hematoxylin and eosin staining for routine histopathological examination. Rabbit polyclonal anti-IGF1 antibody (Abcam, ab9572, Cambridge, USA), reactive against human IGF1, was used in 1:100 dilutions for immunohistochemistry (IHC) as per the manufacturer's details. Fourteen patients with acromegaly and 14 healthy controls were compared for IGF1 immunopositivity. To study the effects of IGF1 in pathological states, IHC was also done in a separate group of 14 cases of $\mathrm{BPH}$ and in 14 cases of prostatic carcinoma of variable Gleason's grade. A slice of liver parenchyma from a 30-year-old healthy individual who underwent autopsy after sudden death following a road traffic accident was taken as positive control. The intensity of IGF1 immunopositivity was expressed on a scale of $0-3$ representing no, mild moderate, or strong positivity (12).

\section{Statistical analysis}

The Statistical Package for the Social Sciences (SPSS) version 16 was used for the statistical analysis. The data were analysed for normality. The continuous variables were analysed using correlation, regression, and paired t-test, while categorical variables were analysed using $\chi^{2}$ test. Nonparametric variables between the control and test groups were analysed using Mann-Whitney $U$ test. Parametric variables between the control and test group were analysed using $t$-test for equality of means. A $P$ value of $<0.05$ was considered as significant.

\section{Results}

Mean age of patients with acromegaly and controls were comparable $(37.9 \pm 9.8$ years vs $41.0 \pm 11.0$ years, $P=0.130)$. Mean GH and IGF1 levels were significantly higher in acromegalics than those in the controls $(P=0.001$; Table 1$)$. Twelve $(22.64 \%)$ patients with acromegaly were treatment naïve, while 41 (77.35\%) had undergone transsphenoidal pituitary surgery by a single neurosurgeon (K K Mukherjee). After the first surgery, two patients underwent trans-frontal surgery, ten received gamma knife, four external beam radiotherapy, and five octreotide LAR for disease control after completion of this study. Thirty-eight (72\%) patients had active acromegaly while 15 (28\%) were cured of their disease.

Mean duration of the disease at presentation was 54 months. In patients who were cured, the median duration of cure was 25 months (3-60 months). Thirtythree of 53 patients with acromegaly were hypogonad and therefore mean serum testosterone was significantly lower in patients with acromegaly compared with controls $(8.9 \pm 8.3 \mathrm{nmol} / \mathrm{l}$ vs $14.3 \pm 3.8 \mathrm{nmol} / \mathrm{l}, P=0.001)$. Among the 33 hypogonad patients, six received testosterone replacement after evaluation.

Patients with acromegaly had significantly higher PSA, higher IPSS, and higher rate of prostatic enlargement on DRE and ultrasound, compared with those in the control group, although all of them had values within normal limits (Table 1). DRE detected prostatic enlargement in a higher number of patients compared with that of ultrasonography. On TRUS, PV was significantly higher in patients with acromegaly $(21.69 \pm 8.66 \mathrm{ml}$ vs $12.48 \pm 3.72 \mathrm{ml}, P=0.001)$. PV $>30 \mathrm{ml}$ was reported in seven patients with acromegaly, but not in controls (13.2\% vs $0 \%, P=0.013)$. Patients who had a PV more than $30 \mathrm{ml}$ had significantly longer duration of disease compared with those who had a volume $<30 \mathrm{ml}$ (96 months vs 44 months, $P=0.023)$. The TR $(4.5 \mathrm{~cm}$ vs $3.6 \mathrm{~cm}$ ) and CC diameters $(3.4 \mathrm{~cm}$ vs $2.3 \mathrm{~cm})$ were also significantly higher in patients with acromegaly compared with controls. 
Table 1 Comparison of various parameters between patients with acromegaly and healthy controls. Data are presented as mean \pm s.D. (range).

\begin{tabular}{l}
\hline Parameters \\
\hline Age (years) \\
GH $(\mathrm{ng} / \mathrm{ml}$ ) nadir after OGTT \\
IGF1 $(\mathrm{ng} / \mathrm{ml})$ \\
Testosterone (nmol/l) \\
PSA (ng/ml) \\
IPSS (0-35) \\
DRE - prostatomegaly \\
USG - prostatic hyperplasia $(\mathrm{PV}>30 \mathrm{ml})$ \\
TRUS (prostate) \\
TR (cm) \\
AP (cm) \\
CC (cm) \\
TV (ml) \\
Median lobe (ml) \\
Cysts \\
Nodules \\
Calcification \\
Uroflowmetry \\
Volume (ml) \\
Maximum flow rate $(\mathrm{ml} / \mathrm{s})$ \\
Average flow rate $(\mathrm{ml} / \mathrm{s})$ \\
PVR (ml)
\end{tabular}

\begin{tabular}{c}
\hline Cases $(n=53)$ \\
$37.9 \pm 9.8(21-60)$ \\
$13.3 \pm 16.4(0.03-76)$ \\
$470.2 \pm 365.6(23.1-1515)$ \\
$8.9 \pm 8.3(0.08-50.0)$ \\
$0.9 \pm 0.7(0.20-2.60)$ \\
$3.9 \pm 3.5(0-21)$ \\
$17(32.1 \%)$ \\
$7(13.2 \%)$ \\
$4.5 \pm 0.7(2.3-6.2)$ \\
$2.7 \pm 0.6(1.5-4.0)$ \\
$3.4 \pm 0.8(1.2-4.8)$ \\
$21.7 \pm 8.7(6.7-49.7)$ \\
$6.0 \pm 3.9(1.5-20)$ \\
$6(11.3 \%)$ \\
$3(5.7 \%)$ \\
$16(30 \%)$ \\
\\
$389.7 \pm 226.5(129-1184)$ \\
$22.4 \pm 11.2(5-63)$ \\
$12.3 \pm 5.5(3-28)$ \\
$20.0 \pm 4.9(0-150)$
\end{tabular}

4

\section{Controls $(n=50)$}

$41.0 \pm 11.0(20-60)$

$0.4 \pm 0.4(0.02-1.70)$

$171.7 \pm 38.6(111-267)$

$14.3 \pm 3.8(10.1-23)$

$0.5 \pm 0.4(0.08-2)$

$0.5 \pm 0.2(0-2)$

$5(10 \%)$

0
$3.6 \pm 0.4(2.3-4.2)$
$2.9 \pm 0.6(1.4-3.7)$
$2.3 \pm 0.6(1.4-4.8)$
$12.5 \pm 3.7(6.3-19.7)$
$2.8 \pm 1.1(2.1-3.6)$
$4(8 \%)$
$1(2 \%)$
$8(16 \%)$

$261.0 \pm 102.6$ (129-688)

$25.3 \pm 5.6(6.20-63)$

$17.4 \pm 3.2(4.5-23)$

$5.3 \pm 7.5(0-22)$

\begin{tabular}{c}
\hline P value \\
\hline 0.130 \\
0.001 \\
0.001 \\
0.001 \\
0.001 \\
0.001 \\
0.008 \\
0.013 \\
\\
0.001 \\
0.057 \\
0.001 \\
0.001 \\
0.216 \\
0.74 \\
0.61 \\
0.10 \\
\\
0.002 \\
0.103 \\
0.001 \\
0.003 \\
\hline
\end{tabular}

OGTT, oral glucose tolerance test; DRE, digital rectal examination; TR, transverse; AP, antero-posterior; CC, cranio-caudal; TV, total volume; PVR, post-void residual volume.

The AP diameter of the prostate was higher in controls, probably a chance observation. Median lobe volume appeared higher in patients with acromegaly $(5.99$ vs $2.85, P=0.216)$. Structural changes in prostate like the occurrence of cysts (11.3\% vs $8 \%$ ), nodules (5.7\% vs $2 \%)$, and calcifications (30\% vs 16\%) were seemingly more common in patients with acromegaly but not significant.

On uroflowmetry, patients with acromegaly showed obstructive features more often than controls. PVR urine volume ranged from 0 to $150 \mathrm{ml}$ in patients with acromegaly, while it was $0-22 \mathrm{ml}$ in controls. PVR more than $50 \mathrm{ml}$ was present in five patients with acromegaly but not in controls (9.4\% vs $0 \%, P=0.001)$.

Fourteen patients underwent prostatic biopsy, of which seven were under 40 years of age. Their IPSS, PV, median lobe volume, and PSA were significantly higher compared with those in controls (Supplementary Table 1). Biopsy showed features of prostatic hyperplasia in six patients $(43 \%)$, stromal inflammation in two (14.28\%), lymphocytic infiltrates in four (28.5\%), and corpora amylacea in four $(28.5 \%)$.

\section{Comparison of prostate parameters in patients with acromegaly below $\mathbf{4 0}$ years of age and controls}

Thirty-one (58.5\%) patients with acromegaly and 24 (48\%) controls were under 40 years of age. Even though serum testosterone was significantly low in young patients with acromegaly (age $<40$ years) compared with age-matched controls, IPSS was significantly higher in these patients (3.87 vs 0 , range $0-21)(P=0.001)$ (Table 2). On TRUS, the TR $(4.37 \mathrm{~cm}$ vs $3.56 \mathrm{~cm}$, $P=0.001)$ and CC $(3.35 \mathrm{~cm}$ vs $2.11 \mathrm{~cm}, P=0.001)$ diameters of the prostate were larger in patients with acromegaly than in controls. The median lobe was measurable only in patients with acromegaly (mean volume $5.22 \mathrm{ml}$, range $1.5-13.80 \mathrm{ml}$ ). PV was higher in patients with acromegaly compared with controls $(20.2 \pm 7.7 \mathrm{ml}$ vs $12.5 \pm 3.7 \mathrm{ml}, P=0.001)$. PV $>30 \mathrm{ml}$ was seen only in patients with acromegaly $(9.67 \%$ vs $0 \%, P=0.248)$. The occurrence of nodules and calcifications was more common in patients with acromegaly. On uroflowmetry, patients with acromegaly showed significant obstructive features in the form of higher PVR urine volume and lower average flow rate. PSA was significantly higher in patients with acromegaly than in controls $(1.00 \mathrm{ng} / \mathrm{ml}$ vs $0.20 \mathrm{ng} / \mathrm{ml}, P<0.001)$ but was in normal range in both the groups (Table 2 ).

The prostate volume (PV) in acromegaly patients with underlying diabetes was not different from those without diabetes. Similarly, the PV does not correlate with fasting plasma insulin, C-peptide, HbA1c, and insulinogenic index (data not shown). 


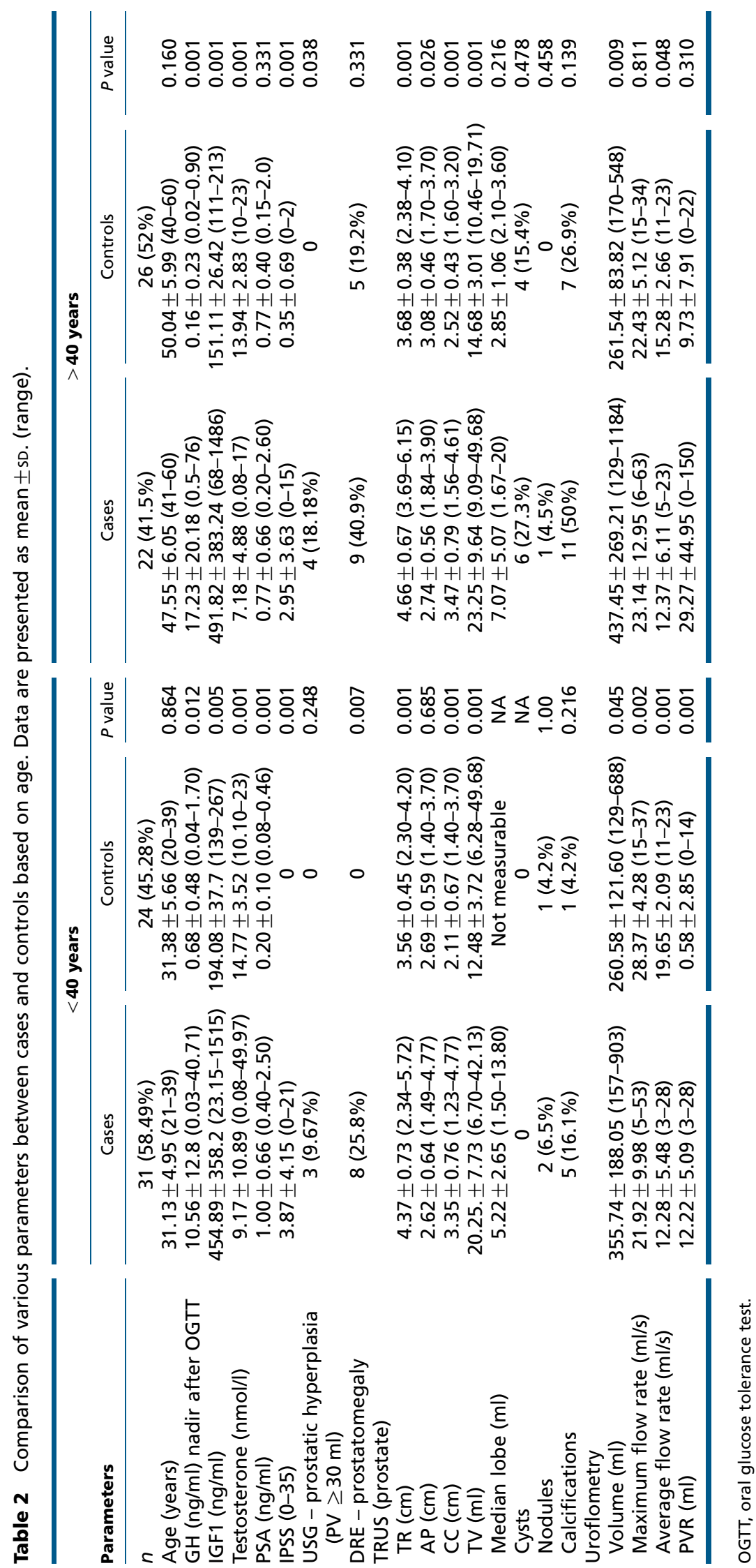




\section{Comparison of prostate parameters in patients with acromegaly older than $\mathbf{4 0}$ years and controls}

In patients of acromegaly older than 40 years, the IPSS was significantly higher compared with controls. Similar trend was found in the median lobe volume, total volume (TV) and PVR of more than $30 \mathrm{ml}$, in patients with acromegaly (Table 2). Structural abnormalities were also more frequently seen in patients. Maximum urine flow rate was marginally higher $(23.1 \mathrm{ml} / \mathrm{s}$ vs $22.4 \mathrm{ml} / \mathrm{s}, P=0.8)$ but the average flow rate was significantly lower (12.4 vs 15.3 , $P=0.05)$ in patients. PVR urine volume was also insignificantly higher in patients compared with controls $(29.3 \mathrm{ml}$ vs $9.4 \mathrm{ml}, P=0.331$ ). Serum PSA levels were similar in both of the groups.

\section{Comparison of prostate parameters in patients with acromegaly: active disease vs inactive disease}

We classified patients with acromegaly on the basis of nadir GH values $<1 \mathrm{ng} / \mathrm{ml}$ on to have inactive disease, or $\geq 1 \mathrm{ng} / \mathrm{ml}$ as active disease. Fifteen (28\%) patients had inactive disease. Age of patients with active and inactive disease was similar. In patients with inactive disease, IPSS was marginally low but did not attain statistical significance $(P=0.81)$, DRE showed prostatic enlargement less often, and prostatic dimensions were less on TRUS. None of the patients with inactive disease, while seven $(18.42 \%$, $P=0.172)$ with active disease had prostatic hyperplasia. Cysts $(5,13.5 \%)$ and calcifications $(14,36.84 \%)$ were more common in those with active disease but nodules were more frequent in those with inactive disease $(13.33 \%$ vs $2.63 \%)$. These structural changes were statistically not significant. Obstructive features were more common in patients with active disease. However, patients with inactive disease still had higher IPSS, PSA level, prostatic dimensions, and obstructive features on uroflowmetry compared with controls (Table 3 and Supplementary Table 2, see section on supplementary data given at the end of this article).

\section{Comparison of prostate parameters in patients with acromegaly: hypogonad vs eugonad}

Based on the serum testosterone levels, patients with acromegaly were classified as hypogonad $(<9 \mathrm{nmol} / \mathrm{l})$ or eugonad ( $>9 \mathrm{nmol} / \mathrm{l})$. IPSS, PV, proportion of patients with PV $>30 \mathrm{ml}$, and obstructive features on uroflowmetry did not differ between these two groups. PSA was comparable in both of the groups (Supplementary Table 3, see section on supplementary data given at the end of this article). Acromegaly patients who were hypogonad had higher IPSS, PSA level, prostatic dimensions, and obstructive features on uroflowmetry compared with controls (Supplementary Table 3). There was no difference in the serum IGF1 levels between patients with BPH and carcinoma prostate $(156.2 \pm 16.2 \mathrm{ng} / \mathrm{ml}$ vs $162.4 \pm 13.2 \mathrm{ng} / \mathrm{ml})$.

\section{Immunohistochemistry}

Of 14 patients with acromegaly who underwent prostate biopsy, eight had active acromegaly and hypogonadism. The mean age of patients with acromegaly and controls were comparable. There were nine subjects aged $<40$ years in both the groups. Six patients with acromegaly had evidence of BPH on biopsy, while none of the controls had BPH (Supplementary Tables 1 and 2). USG was 50\% concordant with histopathology and 83\% with DRE to diagnose BPH. On IHC with anti-IGF1 antibody, the postmortem liver biopsy specimen was strongly positive $(3+)$ (Fig. 1). All patients with acromegaly had moderate positivity $(2+)$ while in controls, three had mild positivity and the rest were negative. BPH tissue showed moderate IHC positivity in two and mild positivity in 12 subjects. As opposed to this, four patients with carcinoma prostate had strong positivity, two each had mild and moderate positivity, while six were negative. The intensity of immunoreactivity to anti-IGF1 antibody positively correlated with Gleason's grade.

\section{Discussion}

Our study demonstrates that patients with acromegaly have higher scores on IPSS, an increased prevalence of PV, obstructive pattern on uroflowmetry, and structural prostatic changes, irrespective of their age, disease activity, or gonadal status. Over-activity of GH/IGF1 axis plays an important role in the pathogenesis of BPH in these patients.

Organomegaly, colonic mucosal hyperplasia, and polyps have been well known in acromegaly as a result of GH-IGF1 over-activity $(13,14,15,16,17,18)$. A few earlier studies have also reported a higher prevalence of prostatic enlargement and BPH in patients with acromegaly $(7,19)$. However, these studies are limited by the lack of histopathological data. Our study is the first to document functional consequences of prostatic enlargement, structural changes by prostatic biopsy, and correlate the pathogenic role of GH/IGF1 axis in these changes.

Prostatic enlargement usually occurs after the age of 40 years, with a prevalence of about $50 \%$ at 50 years and 
Table 3 Comparison of various parameters between acromegaly patients with active and inactive disease compared with healthy controls. Data are presented as mean \pm s.D. (range).

\begin{tabular}{|c|c|c|c|c|c|}
\hline Parameters & Inactive disease & Active disease & $P$ value & Controls & $\begin{array}{l}\boldsymbol{P} \text { value } \\
\text { (inactive vs } \\
\text { control) }\end{array}$ \\
\hline$n$ & $15(28.3 \%)$ & $38(71.69 \%)$ & 0.20 & 50 & \\
\hline Age (years) & $35.20 \pm 9.04$ & $39.03 \pm 9.95$ & 0.202 & $41.0 \pm 11.0(20-60)$ & 0.062 \\
\hline $\begin{array}{l}\mathrm{GH}(\mathrm{ng} / \mathrm{ml}) \text { nadir } \\
\text { after OGTT }\end{array}$ & $0.38 \pm 0.33(0.03-0.97)$ & $18.44 \pm 16.87(1.05-76)$ & 0.001 & $0.4 \pm 0.4(0.02-1.70)$ & 0.72 \\
\hline IGF1 (ng/ml) & $203.30 \pm 152.64(23.15-545)$ & $575.59 \pm 372.52(96-1515)$ & 0.001 & $171.7 \pm 38.6(111-267)$ & 0.94 \\
\hline Testosterone (nmol/l) & $10.38 \pm 13.15(0.08-49.97)$ & $7.54 \pm 6.57(0.16-32)$ & 0.937 & $14.3 \pm 3.8(10.1-23)$ & 0.003 \\
\hline IPSS (0-35) & $3.27 \pm 2.63(0-9)$ & $3.58 \pm 4.37(0-21)$ & 0.810 & $0.5 \pm 0.2(0-2)$ & 0.001 \\
\hline $\begin{array}{l}\text { USG - prostatic hyper- } \\
\text { plasia (PV } \geq 30 \mathrm{ml})\end{array}$ & 0 & $7(18.42 \%)$ & 0.172 & 0 & 0.99 \\
\hline PSA (ng/ml) & $1.18 \pm 0.83(0.20-2.60)$ & $0.80 \pm 0.57(0.2-2.5)$ & 0.022 & $0.5 \pm 0.4(0.08-2)$ & 0.001 \\
\hline DRE - prostatomegaly & $3(20 \%)$ & $14(36.8 \%)$ & 0.333 & $5(10 \%)$ & 0.37 \\
\hline \multicolumn{6}{|l|}{ TRUS (prostate) } \\
\hline $\mathrm{TR}(\mathrm{cm})$ & $4.28 \pm 0.78(2.34-5.72)$ & $4.57 \pm 0.68(2.80-6.15)$ & 0.277 & $3.6 \pm 0.4(2.3-4.2)$ & 0.001 \\
\hline $\mathrm{AP}(\mathrm{cm})$ & $2.46 \pm 0.51(1.77-3.64)$ & $2.75 \pm 0.62(1.49-4.04)$ & 0.088 & $2.9 \pm 0.6(1.4-3.7)$ & 0.005 \\
\hline $\mathrm{CC}(\mathrm{cm})$ & $3.30 \pm 0.60(2-4.20)$ & $3.43 \pm 0.83(1.23-4.77)$ & 0.034 & $2.3 \pm 0.6(1.4-4.8)$ & 0.001 \\
\hline TV (ml) & $17.98 . \pm 5.86(11.70-26.50)$ & $23.16 \pm 9.20(6.70-49.68)$ & 0.04 & $12.5 \pm 3.7(6.3-19.7)$ & 0.001 \\
\hline Median lobe (ml) & $4.84 \pm 2.14(1.76-9.72)$ & $6.44 \pm 4.35(1.50-20)$ & 0.28 & $2.8 \pm 1.1(2.1-3.6)$ & 0.001 \\
\hline Cysts & $1(6.66 \%)$ & $5(13.5 \%)$ & 0.66 & $4(8 \%)$ & 0.99 \\
\hline Nodules & $2(13.33 \%)$ & $1(2.63 \%)$ & 1.00 & $1(2 \%)$ & 0.41 \\
\hline Calcifications & $2(13.33 \%)$ & $14(36.84 \%)$ & 0.11 & $8(16 \%)$ & 0.99 \\
\hline \multicolumn{6}{|l|}{ Uroflowmetry } \\
\hline Volume (ml) & $341 \pm 184.33(194-724)$ & $408 \pm 240(129-1184)$ & 0.33 & $261.0 \pm 102.6(129-688)$ & 0.17 \\
\hline $\begin{array}{l}\text { Maximum flow } \\
\text { rate }(\mathrm{ml} / \mathrm{s})\end{array}$ & $19.89 \pm 7.18(5-32)$ & $23.42 \pm 12.38(6.20-63)$ & 0.63 & $25.3 \pm 5.6(6.20-63)$ & 0.003 \\
\hline $\begin{array}{l}\text { Average flow } \\
\text { rate }(\mathrm{ml} / \mathrm{s})\end{array}$ & $11.38 \pm 4.14(3-20)$ & $12.63 \pm 5.94(4.5-28)$ & 0.74 & $17.4 \pm 3.2(4.5-23)$ & 0.001 \\
\hline PVR (ml) & $14.07 \pm 19.06(0-70)$ & $23.39 \pm 40.58(0-150)$ & 0.79 & $5.3 \pm 7.5(0-22)$ & 0.048 \\
\hline
\end{tabular}

OGTT, oral glucose tolerance test.

$80 \%$ at 80 years (20). Testosterone and DHT play an important role in prostatic cell growth and differentiation; although these hormones are essential, they are not sufficient by themselves (21). Various other growth factors that are involved in growth, development, and pathological enlargement of prostate include epidermal growth factor, fibroblast growth factor, and transforming growth factor beta (22). Little is known about the direct involvement of GH/IGF1 axis in this process. Increased expression of IGF1 receptors (IGF1Rs) has been documented in animal and human models of BPH and prostatic malignancies. Whether the action of circulating or paracrine IGF1 is important at this site is not known $(5,6)$. Recent researches have highlighted the involvement of not only IGF1 signalling but also GH itself. GH is responsible for activation of transcription 5 (STAT5) protein (23). It was shown that STAT5 activation is involved in the development of breast cancer, leukemia, and colon adenocarcinoma as well as prostate cancer. Studies have shown that patients with type 2 diabetes have significantly increased risk of $\mathrm{BPH}$ and the most obvious mechanism involves mitogenic and growthpromoting actions of insulin $(24,25)$. In addition, lower sex hormone-binding globulin associated with insulin resistance is likely to increase the trafficking of testosterone to prostate. The association of $\mathrm{BPH}$ with elevated fasting plasma glucose in men without diabetes is low, suggesting that greater perturbation of glucose homoeostasis is increasingly associated with prostatic enlargement. The lack of correlation of PV with fasting plasma insulin, insulinogenic index, and HbA1c in our study suggests their minimal role in the causation of prostatic enlargement, which is in contrast to a study by Wang et al. (24). It could be explained by fewer acromegaly patients with diabetes in our cohort.

In our study, IPSS was significantly higher in patients with acromegaly compared with controls, even after adjustment of disease status (active or inactive), gonadal status, and age. The IPSS score in patients with acromegaly older than 40 years was significantly higher than in control group, which is distinct from other studies (19). In vitro data suggests that $\mathrm{GH}$ augments the effects of 


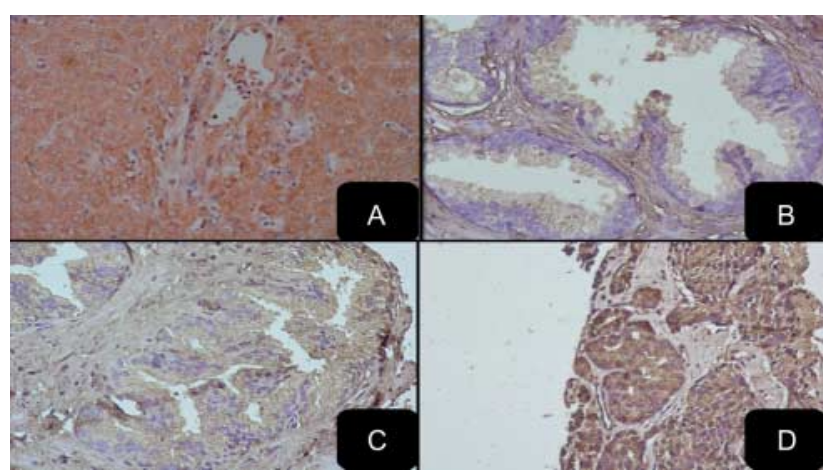

\section{Figure 1}

(A, B, C and D) (400 × magnification) IGF1 immunostaining (granular cytoplasmic staining is considered as positive).

(A) Postmortem adult liver (hepatocytes) taken as positive control (3 + intensity); (B) BPH (1+ intensity); (C) acromegaly $(2+$ intensity); and (D) one of the adenocarcinomas of prostate in a patient $(3+$ intensity).

DHT (26) and inhibits $5 \alpha$-reductase 1 enzyme activity in pilosebaceous unit, thereby reducing the effect of DHT, which is a therapeutic paradox (27). The GHRH antagonists have been shown to reduce the PV directly acting on truncated receptor splice variant without involving the testosterone-DHT pathway (28). Moreover, treatment with $5 \alpha$-reductase inhibitor for BPH or LH-releasing hormone antagonist/agonist for prostate cancer has been shown to reduce prostate size. The literature is silent regarding the activity of $5 \alpha$-reductase 2 in the prostate gland of acromegaly patients. We postulate that elderly subjects already express urinary symptoms but the over-activity of GH/IGF1 axis acts as a 'fuel in the fire' even with bare minimum testosterone and DHT levels and make symptoms more overt in patients with acromegaly. This is evident by IGF1 signalling conferring stimulatory effect on androgenic receptor by attenuating forkhead box-containing protein O subfamily (Foxo1) inhibition (29).

In our study, prostatic enlargement was significantly higher among patients with acromegaly than controls, which is in consonance with other studies $(19,30)$. This was irrespective of age and disease activity or gonadal status in contrast to study reported by Correa et al. (19). Although DRE overestimated prostatic enlargement, compared with USG, there was a good correlation between them $(r=0.9)$. The prevalence of $\mathrm{BPH}$ and structural lesions was more in the study by Colao et al. (30). It could be because of observational bias and lack of histopathological documentation in the above-mentioned study. The lower prevalence of prostatic hyperplasia in our study could be because of the younger population, ethnicity, and histopathological criteria for defining it.

Compared with age-matched controls, the PV was also higher in patients with acromegaly who were hypogonad, although none of them had PV $>30 \mathrm{ml}$. Thus, it could be postulated that the prostatic enlargement not only depends on gonadal axis but also on GH/IGF1 axis. This is further supported by lack of significant prostatic enlargement (PV $>30 \mathrm{ml}$ ) in patients with inactive disease. However, in patients with inactive disease (duration more $\geq 24$ months), PV was still higher than in controls $(20.9 \mathrm{ml}$ vs $12.5 \mathrm{ml}, P=0.001)$. This could be because of the fact that once the prostate is enlarged, it would take a long time for regression, irrespective of the disease control. A previous prospective study by Colao et al. (31) had shown a significant decrease in PV following normalization of GH/IGF1 after 1 year of octreotide treatment. Our study was cross-sectional, which has limitation due to lack of availability of baseline patient characteristics due to which we could not make an appropriate analysis of the effects of treatment(s).

The median lobe of the prostate gland was measurable in all patients with acromegaly, irrespective of their age but only in a few controls older than 40 years. None of the controls younger than 40 years had measurable median lobe. Similarly, the median lobe volume was marginally higher in patients than in controls (5.99 vs $2.85, P=0.216$ ).

Presence of structural changes in prostate such as calcifications, cyst, and nodules was more in patients with acromegaly compared with healthy controls, irrespective of their age. The structural changes found in the prostate of patients with acromegaly in our study were similar to these noted in previous studies $(7,19)$. Prostatic histopathology revealed BPH in six of 14 (43\%) patients. None of the previous studies have performed prostatic biopsy in patients with acromegaly patients to evaluate $\mathrm{BPH}$. Elevated PSA or prostatic carcinomas were not found in any of our patients.

On uroflowmetry, maximum flow rate and average flow rate were reduced in patients with acromegaly and a higher number of patients had significant PVR, which means that there is a dynamic obstruction of lower urinary tract irrespective of the disease activity. Obstructive features were also significantly higher even in younger patients, compared with controls $(P<0.05)$; however, all other parameters except average flow rate were comparable in those older than 40 years, which could be part of the normal ageing process. 
In normal individuals, IGF1 stimulates cell proliferation while its major binding protein (IGFBP3) causes apoptosis. Serum IGF1 in the upper quartile of normal range has been shown to be associated with a higher risk of various neoplasms like carcinoma colon and prostate in the general population (6). In our study, BPH and carcinoma prostate had comparable IGF1 levels to that of healthy controls, even though they were much older and by this time the IGF1 is expected to be low due to somatopause. Published data suggests that BPH as defined by TRUS is well described in patients with acromegaly $(1,19)$. However, occurrence of prostatic carcinoma in this subset of patients is infrequent (8). The physiological development and growth of prostate depends predominantly on testosterone and DHT. Our study has shown the possible involvement of GH/IGF1 axis in the development of BPH in patients with acromegaly and may have some role in the pathogenesis of BPH and prostatic carcinoma in the general population. The variable immune positivity for IGF1 in patients with carcinoma prostate could be because of complex interplay of endocrine and paracrine GH/IGF1 and the binding proteins, variable IGF1R expression, or involvement of unidentified pathways. IGF1R expression is seen in both hormone responsive and castrated hormone refractory prostatic cancer and $\mathrm{BPH}$ specimens. Activation of androgen receptor via interaction of IGF1 and IGF2 through IGF1R is one of the putative mechanisms in these disorders (32). In six patients with inactive disease, the moderate positivity for anti-IGF1 antibody is not surprising as there is a dissociation between serum and tissue IGF1 level, and once the process of BPH has started, it may take a longer time to regress or may not regress at all. In future studies, the IGF1R status should be examined in prostatic biopsy specimen of acromegaly patients.

The strengths of our study include the presence of an age- and BMI-matched control group, prostatic biopsy, and IHC for anti-IGF1 antibody; however, the limitations are small sample size, cross-sectional nature of the study, and lack of long-term follow-up to look for recovery of prostatic enlargement after different therapies. It is still uncertain whether prostate disorders may be a clinical concern in patients with acromegaly, since in clinical practice very few patients complain of symptoms related to prostatic hyperplasia or neoplasia.

\section{Conclusion}

In patients with acromegaly, there is a higher frequency of structural changes in prostate, along with a greater PV and obstructive features compared with healthy controls, irrespective of age, gonadal status, or disease activity but the differences in various parameters between acromegaly patients and controls seem to be marginal. These findings indicate that very few of the patients had clinically relevant or significant disease. Therefore, a careful prostatic symptom evaluation, supported by TRUS, may not be recommended in the work-up of all male patients with acromegaly. Future prospective multicenter studies on larger populations of acromegaly patients may clarify this aspect.

\section{Supplementary data}

This is linked to the online version of the paper at http://dx.doi.org/10.1530/ EJE-14-0698.

\section{Declaration of interest}

The authors declare that there is no conflict of interest that could be perceived as prejudicing the impartiality of the research reported.

\section{Funding}

This research did not receive any specific grant from any funding agency in the public, commercial or not-for-profit sector.

\section{Acknowledgements}

We are very grateful to the patients who consented to participate in this study. We are thankful to Prof. R J Dash (Emeritus Professor and Head of Department of Endocrinology, PGIMER, Chandigarh) and Dr R Muralidharan (Formerly Associate Professor, Department of Endocrinology, PGIMER, Chandigarh) for their constructive comments and manuscript editing We also thank Cipla India Ltd for the generous gift of anti-IGF1 antibody. We would like to thank Dr Anupam Lal, Department of Radiodiagnosis, PGIMER, Chandigarh for helping us with ultrasonography.

\section{References}

1 McNeal JE. Normal histology of the prostate. American Journal of Surgical Pathology 198812 619-633. (doi:10.1097/00000478-198808000-00003)

2 Wilson JD. The pathogenesis of benign prostatic hyperplasia. American Journal of Medicine 198068 745-756. (doi:10.1016/00029343(80)90267-3)

3 Melmed S. Acromegaly. New England Journal of Medicine 1990322 966-977. (doi:10.1056/NEJM199004053221405)

4 Chan JM, Stampfer MJ, Giovannucci E, Gann PH, Ma J, Wilkinson P, Hennekens $\mathrm{CH} \&$ Pollak M. Plasma insulin-like growth factor-1 and prostate cancer risk: a prospective study. Science 1998279 563-566. (doi:10.1126/science.279.5350.563)

5 Wolk A, Mantzoros CS, Andersson SO, Bergström H, Signorello LB, Lagiou P, Adami HO \& Trichopoulos D. Tri insulin-like growth factor 1 and prostate cancer risk: a population-based, case-control study. Journal of the National Cancer Institute 199890 911-915. (doi:10.1093/ jnci/90.12.911)

6 Mantzoros CS, Tzonou A, Signorello LB, Stampfer M, Trichopoulos D \& Adami HO. Insulin-like growth factor 1 in relation to prostate cancer and benign prostatic hyperplasia. British Journal of Cancer $1997 \mathbf{7 6}$ 1115-1118. (doi:10.1038/bjc.1997.520) 
7 Colao A, Marzullo P, Spiezia S, Ferone D, Giaccio A, Cerbone G, Pivonello R, Di Somma C \& Lombardi G. Effect of growth hormone (GH) and insulin-like growth factor I on prostatic diseases: an ultrasonographic and endocrine study in acromegaly, GH deficiency, and healthy subjects. Journal of Clinical Endocrinology and Metabolism 199984 1986-1991. (doi:10.1210/jcem.84.6.5776)

8 Colao A, Marzullo P, Spiezia S \& Lombardi G. Acromegaly and prostate cancer. Growth Hormone \& IGF Research 200010 (Suppl A) S37-S38. (doi:10.1016/S1096-6374(00)90018-2)

9 Collins GN, Raab GM, Hehir M, King B \& Garraway WM. Reproducibility and observer variability of transrectal ultrasound measurements of prostatic volume. Ultrasound in Medicine \& Biology 199521 1101-1105. (doi:10.1016/0301-5629(95)02001-2)

10 Barry MJ, Fowler FJ Jr, O’Leary MP, Bruskewitz RC, Holtgrewe HL Mebust WK \& Cockett AT. The American Urological Association symptom index for benign prostatic hyperplasia. The Measurement Committee of the American Urological Association. Journal of Urology 1992148 1549-1557.

11 Bosch JL, Hop WC, Kirkels WJ \& Schröder FH. The International Prostate Symptom Score in a community-based sample of men between 55 and 74 years of age: prevalence and correlation of symptoms with age, prostate volume, flow rate and residual urine volume. British Journal of Urology 199575 622-630. (doi:10.1111/j.1464-410X.1995.tb07421.x)

12 Lidhar K, Korbonits M, Jordan S, Khalimova Z, Kaltsas G, Lu X, Clayton RN, Jenkins PJ, Monson JP, Besser GM et al. Low expression of the cell cycle inhibitor p27Kip1 in normal corticotroph cells, corticotroph tumors, and malignant pituitary tumors. Journal of Clinical Endocrinology and Metabolism 199984 3823-3830. (doi:10.1210/jcem.84.10.6066)

13 Renehan AG, Bhaskar P, Painter JE, O'Dwyer ST, Haboubi N, Varma J, Ball SG \& Shalet SM. The prevalence and characteristics of colorectal neoplasia in acromegaly. Journal of Clinical Endocrinology and Metabolism 200085 3417-3424. (doi:10.1210/jcem.85.9.6775)

14 Bogazzi F, Cosci C, Sardella C, Costa A, Manetti L, Gasperi M, Rossi G, Bartalena $\mathrm{L} \&$ Martino E. Identification of acromegalic patients at risk of developing colonic adenomas. Journal of Clinical Endocrinology and Metabolism 200691 1351-1356. (doi:10.1210/jc.2005-2500)

15 Terzolo M \& Boccuzzi A. The prevalence of colonic polyps in acromegaly: a colonoscopic and pathological study in 103 patients. Journal of Clinical Endocrinology and Metabolism 199681 2406-2407. (doi:10.1210/jcem.81.6.8964888)

16 Colao A, Pivonello R, Auriemma RS, Galdiero M, Ferone D, Minuto F, Marzullo P \& Lombardi G. The association of fasting insulin concentrations and colonic neoplasms in acromegaly: a colonoscopybased study in 210 patients. Journal of Clinical Endocrinology and Metabolism 200792 3854-3860. (doi:10.1210/jc.2006-2551)

17 Colao A, Ferone D, Marzullo P \& Lombardi G. Systemic complications of acromegaly: epidemiology, pathogenesis, and management. Endocrine Reviews 200425 102-152. (doi:10.1210/er.2002-0022)

18 Delhougne B, Deneux C, Abs R, Chanson P, Fierens H, Laurent-Puig P, Duysburgh I, Stevenaert A, Tabarin A, Delwaide J et al. The prevalence of colonic polyps in acromegaly: a colonoscopic and pathological study in 103 patients. Journal of Clinical Endocrinology and Metabolism 199580 3223-3226. (doi:10.1210/jcem.80.11.7593429)
19 Correa LL, Balarini Lima GA, Cavallieri SA, Miranda LC \& Gadelha MR. Prostatic disorders in a acromegalic patients experience of a Brazilian center. International Brazilian Journal of Urology 201339 393-401. (doi:10.1590/S1677-5538.IBJU.2013.03.13)

20 Berry SJ, Coffey DS, Walsh PC \& Ewing LL. The development of human benign prostatic hyperplasia with age. Journal of Urology 1984132 474-479.

21 Motta M, Dondi D, Moretti RM, Montagnani Marelli M, Pimpinelli F, Maggi R \& Limonta P. Role of growth factors, steroid and peptide hormones in the regulation of human prostatic tumor growth. Journal of Steroid Biochemistry and Molecular Biology 199656 107-111. (doi:10.1016/0960-0760(95)00240-5)

22 Cunha GR, Donjacour AA, Cooke PS, Mee S, Bigsby RM, Higgins SJ \& Sugimura $Y$. The endocrinology and developmental biology of the prostate. Endocrine Reviews 19878 338-362. (doi:10.1210/edrv-8-3-338)

23 Levy DE \& Gilliland DG. Divergent roles of STAT1 and STAT5 in malignancy as revealed by gene disruptions in mice. Oncogene 200019 2505-2510. (doi:10.1038/sj.onc.1203480)

24 Wang Z \& Olumi AF. Diabetes, growth hormone-insulin-like growth factor pathways and association to benign prostatic hyperplasia. Differentiation 201182 261-271. (doi:10.1016/j.diff.2011.04.004)

25 Parsons JK, Carter HB, Partin AW, Windham BG, Metter EJ, Ferrucci L, Landis P \& Platz EA. Metabolic factors associated with benign prostatic hyperplasia. Journal of Clinical Endocrinology and Metabolism 200691 2562-2568. (doi:10.1210/jc.2005-2799)

26 Deplewski D \& Rosenfield RL. Growth hormone and insulin-like growth factors have different effects on sebaceous cell growth and differentiation. Endocrinology 1999140 4089-4094. (doi:10.1210/endo. 140.9.6957)

27 Trüeb RM. Hormones and hair growth. Der Hautarzt 201061 487-495. (doi:10.1007/s00105-009-1890-2)

28 Rick FG, Schally AV, Block NL, Nadji M, Szepeshazi K, Zarandi M, Vidaurre I, Perez R, Halmos G \& Szalontay L. Antagonists of growth hormone-releasing hormone (GHRH) reduce prostate size in experimental benign prostatic hyperplasia. PNAS $20111083755-3760$. (doi:10.1073/pnas.1018086108)

29 Yanase T \& Fan W. Modification of androgen receptor function by IGF-1 signaling implication in the mechanism of refractory prostate carcinoma. Vitamins and Hormones 200980 649-666. (doi:10.1016/ S0083-6729(08)00623-7)

30 Colao A, Marzullo P, Ferone D, Spiezia S, Cerbone G, Marinò V, Di Sarno A, Merola B \& Lombardi G. Prostatic hyperplasia: an unknown feature of acromegaly. Journal of Clinical Endocrinology and Metabolism 199883 775-779. (doi:10.1210/jcem.83.3.4645)

31 Colao A, Marzullo P, Spiezia S, Giaccio A, Ferone D, Cerbone G, Di Sarno A \& Lombardi G. Effect of two years of growth hormone and insulin-like growth factor-I suppression on prostate diseases in acromegalic patients. Journal of Clinical Endocrinology and Metabolism 200085 3754-3761. (doi:10.1210/jcem.85.10.6907)

32 Ryan CJ, Haqq CM, Simko J, Nonaka DF, Chan JM, Weinberg V, Small EJ \& Goldfine ID. Expression of insulin like growth factor-1 receptor in local and metastatic prostate cancer. Urologic Oncology 2007 25 134-140. (doi:10.1016/j.urolonc.2006.07.019)

Received 13 August 2014

Revised version received 17 October 2014

Accepted 6 November 2014 